\title{
Analysis of Sound Absorption Performance of Underwater Acoustic Coating considering Different Poisson's Ratio Values
}

\author{
Zhuhui Luo $\mathbb{D}^{1},{ }^{1}$ Tao Li, ${ }^{1}$ Yuanwei Yan, ${ }^{2}$ and Zhou Zhou ${ }^{2}$ \\ ${ }^{1}$ School of Mechanical Engineering, Hunan University of Technology, Zhuzhou, China \\ ${ }^{2}$ Zhuzhou Times New Materials Technology CO,.LTD, Zhuzhou, China \\ Correspondence should be addressed to Zhuhui Luo; 10925022@zju.edu.cn
}

Received 2 August 2021; Revised 2 September 2021; Accepted 4 September 2021; Published 24 September 2021

Academic Editor: Azwan I. Azmi

Copyright (c) 2021 Zhuhui Luo et al. This is an open access article distributed under the Creative Commons Attribution License, which permits unrestricted use, distribution, and reproduction in any medium, provided the original work is properly cited.

Viscoelastic material acoustic coating plays an important role in noise and vibration control of underwater equipment. The dynamic mechanical properties of the viscoelastic material have a direct effect on the sound absorption performance of the acoustic coating. The influence of Poisson's ratio on sound absorption performance is studied. A finite element model was established to calculate the sound absorption performance of three typical acoustic coatings: homogeneous acoustic coatings, Alberich acoustic coatings, and trumpet cavity acoustic coatings, and the influence of Poisson's ratio on the sound absorption performance of the three kinds of acoustic coatings was analyzed. The results show that when Poisson's ratio varies from 0.49 to 0.4999, the larger Poisson's ratio is, the larger the frequency of the first absorption peak is, the smaller the absorption coefficient below the frequency of the first absorption peak is, and the smaller the average absorption coefficient in the whole analysis frequency range is. The dynamic Poisson's ratio with the change of frequency is obtained by interpolating the test results and static Poisson's ratio finite element calculation results. The calculation results show that the dynamic Poisson's ratio can get more accurate calculation results. This work can provide a reference for researchers to set Poisson's ratio in theoretical analysis and finite element analysis of acoustic coating.

\section{Introduction}

Acoustic coatings of viscoelastic materials play an important role in noise and vibration control of underwater equipment. The typical acoustic coating structure used in underwater equipment is homogeneous acoustic coating $[1,2]$, Alberich acoustic coating [3, 4], and trumpet cavity-type coating [5]. Homogeneous acoustic coating has the characteristics of simple structure, convenient installation and maintenance, and low cost, but its low-frequency sound absorption performance is poor. Alberich coating originated in Germany during World War II and has excellent low-frequency sound absorption performance. The structure of trumpet cavitytype coating is more complex, and the low-frequency sound absorption performance is also excellent. Alberich acoustic coating and trumpet cavity-type coating belong to resonant cavity acoustic coating. According to these two acoustic coating, various forms of resonant cavity acoustic coating are derived. Sound absorption performance is one of the most important properties of acoustic coatings [6]. In addition to structure, dynamic mechanical property parameters of materials used in acoustic coatings have a direct impact on sound absorption performance $[7,8]$. There are six parameters to describe the dynamic mechanical properties of viscoelastic materials: Young's modulus, shear model, Poisson's ratio, Lamy constant, bulk compression modulus, and bulk longitudinal wave modulus. Among the six parameters, only two parameters are independent; among which Young's modulus and Poisson's ratio are the two commonly used parameters $[9,10]$. Either through frequency expansion or through inversely solving acoustic measurement such as sound velocity, the Young modulus of viscoelastic materials can be obtained. Defined as the negative value of the ratio of the transverse positive strain to the axial positive strain when a material is under unidirectional tension or compression, Poisson's ratio can be obtained 
either through measurement or calculation with the Young modulus, shear modulus, or bulk modulus. Regardless, the order of magnitude for the accuracy of Poisson's ratio obtained can reach only $0.01[11,12]$.

The influence of Poisson's ratio on the acoustic performance of coatings is seldom studied. Since viscoelastic materials are approximately incompressible materials, Poisson's ratio is difficult to be measured with high precision (the measurement accuracy is generally 0.01). According to this actual situation, researchers usually assume Poisson's ratio to be a value close to but less than 0.5 such as 0.49 [9, 13-20], 0.493 [21], 0.495 [15, 19], 0.4997 [19], 0.49976 [15], and 0.499981 [22]. It can be seen that Poisson's ratio of viscoelastic materials is set differently in the above literatures (although the difference in value is very small). This paper is to study whether and what effects such slight difference has on sound absorption performance. In addition, in these literatures, Poisson's ratio is set as a static value that does not change with the frequency. In literature [13], when studying the influence of Poisson's loss factor on acoustic covering layer, the real part of Poisson's ratio is still regarded as a fixed value. In fact, Poisson's ratio of viscoelastic materials varies with frequency. In this paper, the finite element calculation of sound absorption performance of acoustic coating is carried out, and Poisson's ratio varying with frequency is used in order to obtain more accurate calculation results.

\section{Poisson's Ratio and the Attenuation Constant of Acoustic Waves}

The Young modulus and Poisson's ratio, two parameters required to describe the properties of viscoelastic materials, are denoted as $E$ and $\nu$. Then, the shear modulus is indicated by $G$, the Lamy constant is denoted as $\lambda$, and the bulk longitudinal wave modulus is represented by $S$.

$$
\begin{aligned}
G & =\frac{E}{2(1+\nu)}, \\
\lambda & =\frac{E \nu}{(1+\nu)(1-2 \nu)}, \\
S & =\lambda+2 G .
\end{aligned}
$$

For the viscoelastic material, both Young's modulus and Poisson's ratio are complex numbers, as a result of which they are the related parameters. In an isotropic infinite medium, the wave velocity of longitudinal waves $c_{l}$ and the wave velocity of shear waves $c_{t}$ are written as follows:

$$
\begin{gathered}
c_{l}=\sqrt{\frac{S}{\rho},} \\
c_{t}=\sqrt{\frac{G}{\rho}},
\end{gathered}
$$

where $\rho$ is the density of the sound wave propagation material. According to $\rho$ and sound velocity $c$, the characteristic impedance of the material is expressed as follows:

$$
Z=\rho c .
$$

The attenuation constant of longitudinal and shear waves in the viscoelastic material $\alpha_{l}$ and $\alpha_{t}$ is expressed as follows:

$$
\begin{aligned}
& \alpha_{l}=\frac{\omega}{2 c_{l}} \eta, \\
& \alpha_{t}=\frac{\omega}{2 c_{t}} \eta,
\end{aligned}
$$

where $\omega$ represents the angular frequency of the sound wave and $\eta$ represents the loss factor of the material. For such viscoelastic materials as rubber, Poisson's ratio approaches 0.5 , and $1-2 v$ is close to 0 . According to Equations (2-8), even a slight change in Poisson's ratio will have a significant impact on the sound velocity of the longitudinal wave, thus affecting the characteristic impedance and attenuation constant of the material and then on the characteristics of the acoustic coating in respect of sound absorption. To some extent, the attenuation constant can characterize only the characteristics of the material in terms of sound absorption. In response to the acoustic coating with a limited size but complex structure, it is necessary to conduct quantitative analysis of the impact caused by Poisson's ratio on the characteristics of sound absorption.

\section{Acoustic Coating}

The acoustic coatings analyzed in this paper are three types of commonly used underwater equipment, including homogeneous coating, Alberich acoustic coating, and trumpet cavity-type coating. Homogeneous coating is an equal thickness coating that does not contain any cavity structure. When in use, it is made into a rectangular block structure and pasted on the outer surface of underwater equipment. Figure 1 shows the underwater structure of the Alberich coating applied. In aquatic environment, acoustic waves are incident perpendicularly along the $-\mathrm{z}$ axis to the coating laid on a steel plate with air behind. Made of viscoelastic material, the acoustic coating has cylindrical cavities uniformly and periodically distributed inside. Statistically, the periodic spacing $L_{1}$ in the $X$ and $Y$ directions is $30 \mathrm{~mm}$, the diameter of the cavities denoted as $d$ is $10 \mathrm{~mm}$, the height of the cylindrical cavities represented by $t_{2}$ is $46 \mathrm{~mm}$, the thickness of the hole sealing layers on both sides of the cavities indicated by $t_{1}$ and $t_{3}$, respectively, is $2 \mathrm{~mm}$, and the total thickness of the coating is $50 \mathrm{~mm}$.

Figure 2 shows the underwater structure of the trumpet cavity-type coating. In aquatic environment, acoustic waves are incident perpendicularly along the $-z$ axis to the coating laid on a steel plate with air behind. Made of a viscoelastic material, the acoustic coating with a shape of trumpet cavity is periodically distributed in the $X$ and $Y$ directions. The cavities are distributed in the form of an equilateral triangle. The side length $L_{2}$ of the equilateral triangle is $14.74 \mathrm{~mm}$. Denoted as $d_{2}$, the diameter of the trumpet with a shape of cavity tube is $1.3 \mathrm{~mm}$. Referred to as $d_{3}$, the diameter of the trumpet mouth is $11 \mathrm{~mm}$. Indicated by $t_{5}$, the height of the cavities is $46 \mathrm{~mm}$. Denoted as $t_{4}$ and $t_{6}$, respectively, the 

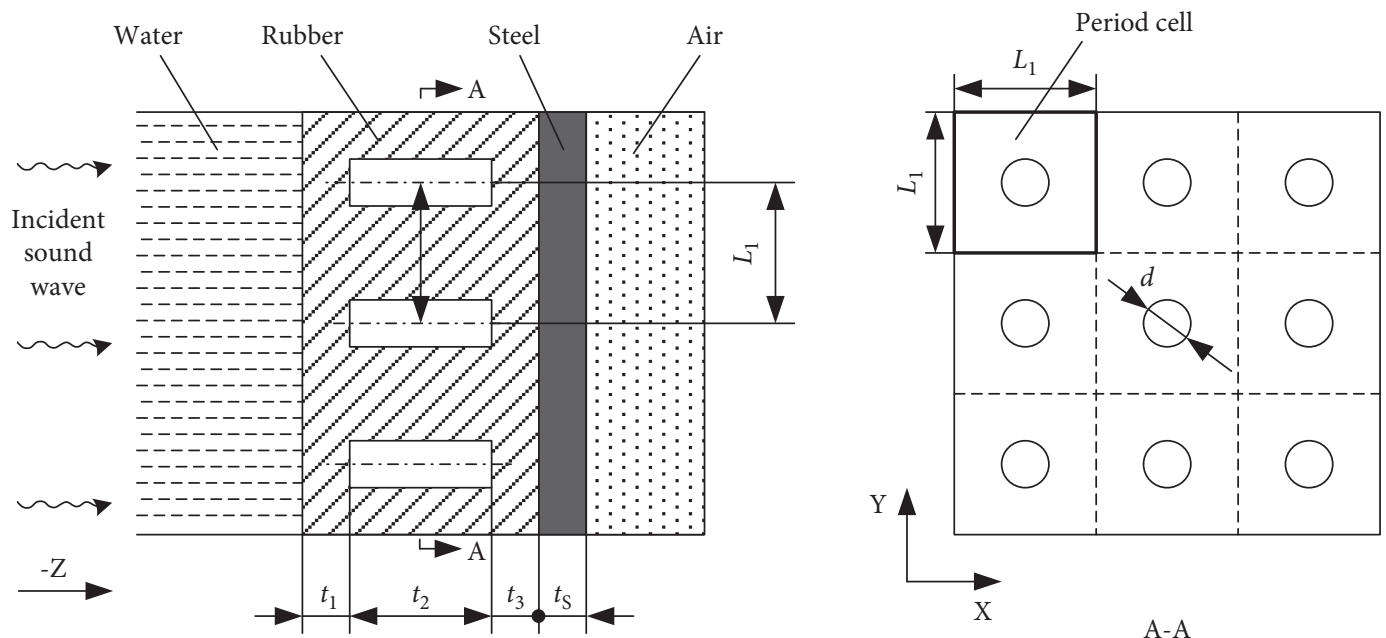

FIgURE 1: Alberich acoustic coating structure.

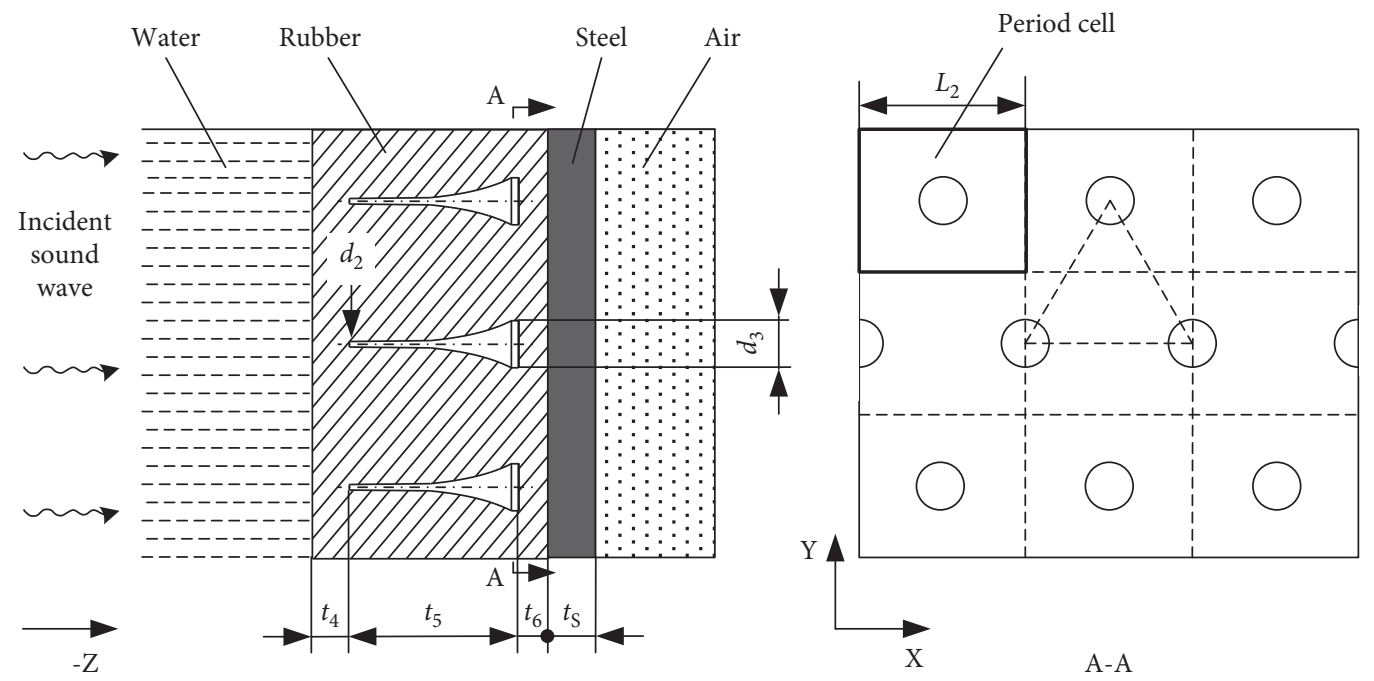

FiguRE 2: Structure of trumpet cavity-type coating.

thickness of the hole sealing layers on both sides of the cavities is $2 \mathrm{~mm}$. The total thickness of the coating is $50 \mathrm{~mm}$.

\section{Finite Element Model}

The finite element method is a very effective method to analyze the sound absorption performance of acoustic coating. Since the 1990s, Hennion and Easwaran [23] began to study acoustic coatings by the finite element method (FEM). Nowadays, commercial numerical simulation software (for example, ANSYS [24], Virtual. Lab [25], and COMSOL $[26,27]$ ) can analyze the acoustic performance of acoustic coatings on complex structures. The finite element modules of the acoustic simulation software LMS Virtual. Lab 11 were applied to construct the finite element model for the sound absorption performance of acoustic coatings, as shown in Figure 3. This problem belongs to acoustic fluid structure coupling problem. The following assumptions are made; that is, water is a homogeneous fluid without flow and viscosity, and different adjacent solids are completely connected. Under the Blouch periodic boundary conditions [26], the periodic unit shown in Figures 1 and 2 was selected for calculation. The finite element model is comprised of water, acoustic coating, and steel plate backing. There is no need to model the air behind the steel plate. The default setting of the software is a total reflection boundary. The grids of the three materials were divided into tetrahedral mesh, with the size of the mesh varying from one structure of the coatings to another. The maximal side lengths of tetrahedral meshes of water, acoustic coating, and steel plate are $2.3 \mathrm{~mm}, 1 \mathrm{~mm}$, and $1 \mathrm{~mm}$, respectively. Therefore, the number of tetrahedral elements of Alberich acoustic coating and trumpet cavity-type coating is 351464 and 74965, respectively. Conode processing was conducted on the surface of contact between the coating and steel plate. Denoted as S1, S2, S3, and S4, respectively, the four sides of the coating and the steel plate were constrained by two degrees of freedom in the $X$ and $Y$ directions. Plane acoustic wave excitation was applied to one end of the water grid. Field point 1 and field point 2 were added in the water meshers, the distance 


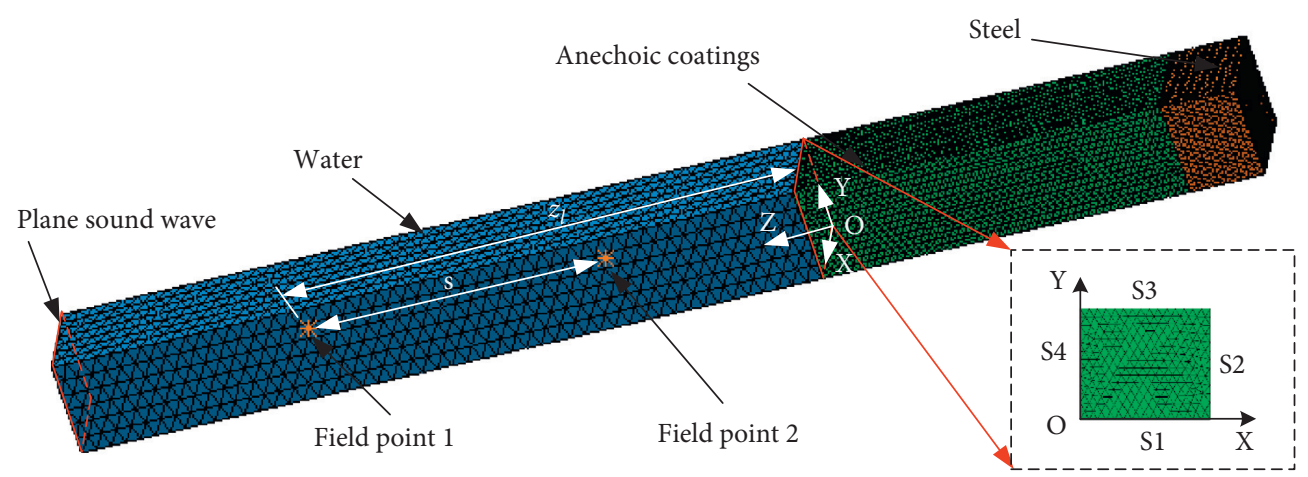

FIGURE 3: Finite element model.

between the two field points is denoted as $s$ and the distance between field point 1 and interface between water and acoustic coating is denoted as $z_{l}$.

The sound pressure of incident and reflected waves in water can be expressed as follows:

$$
\begin{aligned}
& p_{i}=P_{I} e^{j k z}, \\
& p_{r}=P_{R} e^{-j k z},
\end{aligned}
$$

where $k$ represents the wave number; $P_{I}$ and $P_{R}$ refer to the amplitudes of the incident wave $p_{i}$ and reflected wave $p_{r}$ on the XOY plane of the datum plane, respectively; $P_{R}=r P_{I}$; and $r$ stands for the reflection coefficient.

Therefore, the sound pressure at the two field points is the superposition of the incident and the reflected waves. Then,

$$
\begin{aligned}
& p_{1}=P_{I} e^{j k z_{l}}+P_{R} e^{-j k z_{l}}, \\
& p_{2}=P_{I} e^{j k\left(z_{l}-s\right)}+P_{R} e^{-j k\left(z_{l}-s\right)} .
\end{aligned}
$$

The transfer function from field point 1 to field point 2 of the total sound field is written as follows:

$$
H_{12}=\frac{p_{2}}{p_{1}}=\frac{P_{I} e^{j k\left(z_{l}-s\right)}+r P_{I} e^{-j k\left(z_{l}-s\right)}}{P_{I} e^{j k z_{l}}+r P_{I} e^{-j k z_{l}}} .
$$

The reflection coefficient $r$ can be obtained using

$$
r=\frac{p_{2} / p_{1}-e^{-j k s}}{e^{j k s}-p_{2} / p_{1}} e^{j 2 k x_{l}} .
$$

During the process of simulation calculation, the sound pressure $p_{1}$ and $p_{2}$ of field point 1 and 2 could be obtained. With $s$ and $z_{l}$ used to obtain the reflection coefficient $r$, the sound absorption coefficient is expressed as

$$
\alpha=1-r^{2} \text {. }
$$

In order to verify the accuracy of the calculation results of the finite element model, a $50 \mathrm{~mm}$ thick homogeneous coating was selected from Ref. [13] for calculation. The geometric parameters and material parameters of the coating are consistent with the literature. That is to say, the Young modulus of rubber is $E=140 \mathrm{MPa}$, the loss factor is 0.23 , the density is $1100 \mathrm{~kg} / \mathrm{m}^{3}$, Poisson's ratio is 0.49 , and 0 is taken as Poisson's loss factor. The Young modulus of the steel plate is $2.16 \times 10^{5} \mathrm{MPa}$, Poisson's ratio is 0.3 , and the density is $7800 \mathrm{~kg} / \mathrm{m}^{3}$. The density of water is $1000 \mathrm{~kg} / \mathrm{m}^{3}$, and the velocity of sound is $1489 \mathrm{~m} / \mathrm{s}$. The above values are used for the material properties of water and steel plate later.

We performed a mesh sensitivity analysis to represent the mesh dependency of the current problem. The side length of tetrahedral meshers of water acoustic coating and steel plate is set to $1 \mathrm{~mm}, 2 \mathrm{~mm}, 4 \mathrm{~mm}$, and $6 \mathrm{~mm}$, and the calculation results of sound absorption coefficient are observed. The results show that the water mesh size has no effect on the results (the graphical results are not given in the paper). Figure 4 shows the comparison drawn between the sound absorption coefficient calculated using the finite element model under different mesh size settings and the calculation results shown in Ref. [13]. The percentage errors between the current results and Ref. [13] are 2.3\%, 2.9\%, $9.5 \%$, and $11.6 \%$. According to the results, when the mesh size is set to $1 \mathrm{~mm}$ and $2 \mathrm{~mm}$, the sound absorption coefficient calculation results are basically consistent with those in Ref. [13], indicating the feasibility of applying the finite element model to conduct research on the setting of Poisson's ratio.

\section{The Impact of Poisson's Ratios on the Performance of Acoustic Coatings in Sound Absorption}

In Section 3, the abovementioned finite element model is adopted to calculate the performance of the three acoustic coatings in sound absorption. In the model, Poisson's ratio is set to $0.490,0.493,0.496,0.499$, and 0.4999 according to the approximate incompressible characteristics of viscoelastic materials and the values commonly used for research. Similar to the modulus, Poisson's ratio is a complex number. In the case of lower analytical frequency than $5000 \mathrm{~Hz}$, however, the imaginary part of Poisson's ratio would be significantly smaller than the real part, indicating that Poisson's loss factor is as insignificant as its impact on the characteristics of sound absorption exhibited by various types of acoustic coatings that can be ignored. Moreover, for most acoustic coatings, the sound absorption coefficients are close to 1 if the analysis frequency exceeds $5000 \mathrm{~Hz}$. Thus, the 


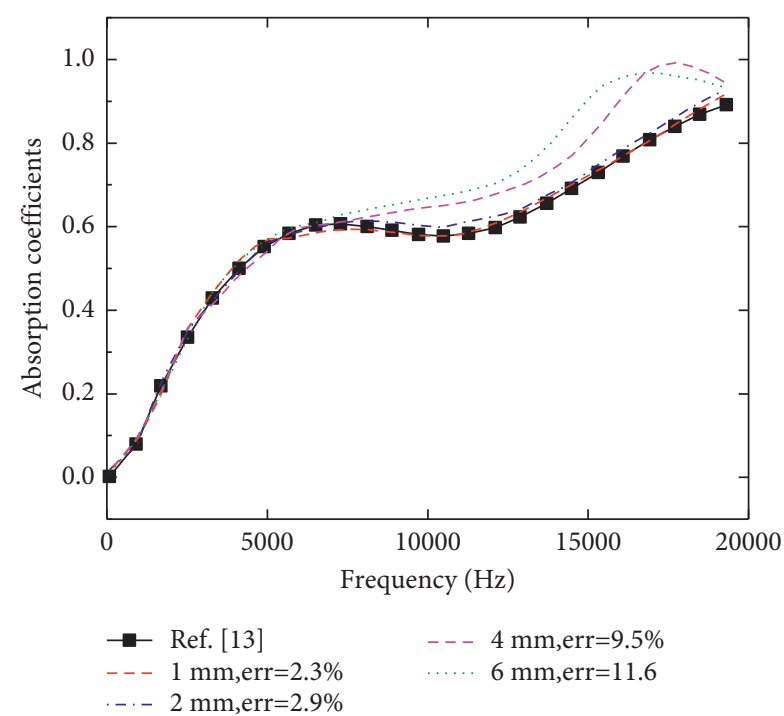

FIgURE 4: Comparison of calculation results in this paper and Ref. [13].

analytical frequency used in this paper ranges from 100 to $5000 \mathrm{~Hz}$, and Poisson's ratio could be set as a real number.

For simplicity purpose, the modulus of materials was set in some research studies to a constant regardless of frequency for the analysis and calculation of the performance of acoustic coatings in sound absorption. In fact, however, it varies with frequency.

As shown in Figure 5, the static modulus is assigned with the value shown in Ref. [13] so that $E=1.4 \times 10^{8} \mathrm{~Pa}$ and the loss factor is 0.23 . Figure 5 shows the results of finite element calculation for the sound absorption coefficients of the three coatings.

Figure 6 shows the sound absorption coefficients of the three coatings as obtained when the modulus of the material is assigned with the modulus parameter of the carbon black filled nitrile rubber shown in Figure 7.

According to Figures 5 and 6, with the increase in Poisson's ratio, the first peak of the sound absorption coefficient curve shifts to high frequency. As for the homogeneous acoustic coating, the same conclusion can be drawn despite no appearance of the sound absorption peak in the figure. The first peak of sound absorption shifts to low frequency, which causes the sound absorption coefficient to decline with the rise of Poisson's ratio when the frequency falls below the peak. This is because the velocity of longitudinal sound increases and the attenuation constant decreases with the rise of Poisson's ratio, as a result of which the sound absorption coefficient of the acoustic coating drops, as shown in Equations (1)-(8) in Section 2.

In figures 5(b), 5(c), 6(b), and 6(c), when Poisson's ratio is 0.499 and 0.4999 , the characteristics of sound absorption coefficient curve are quite different from those when Poisson's ratio is $0.490,0.493$, and 0.496 , which may be due to the high sensitivity of sound absorption coefficient to Poisson's ratio. As shown in Equations (3), Poisson's ratio is close to 0.5 , and the denominator in the calculation formula of the Lamy constant tends to 0. A small change in Poisson's ratio can cause a great change in the Lamy constant, resulting in a large change in sound absorption coefficient. Table 1 shows the percentage of change rate of Poisson's ratio and the percentage of change rate of the Lamy constant when Young's modulus is $140 \mathrm{MPa}$. It can be seen that when Poisson's ratio is 0.493 and 0.496 , the change rate of the Lamy constant is relatively small, while when Poisson's ratio is 0.499 and 0.4999 , the change rate of the Lamy constant is very large. Therefore, when Poisson's ratio is 0.499 and 0.4999 , the sound absorption coefficient curve is quite different.

In order to further analyze the influence of Poisson's ratios on the sound absorption coefficient, the modulus was assigned with the static value and dynamic value. Figure 8 shows the average sound absorption coefficient of the three coatings ranging from 100 to $5000 \mathrm{~Hz}$. As shown in the figure, the average value of sound absorption decreases with the increase in Poisson's ratio regardless of whether the modulus value is static or dynamic and what type of acoustic coating it is. Besides, it can be seen from Figure 8 that the homogeneous one has different characteristics to the other two acoustic coatings as manifested mainly in its smaller average sound absorption coefficient, and the pattern of coefficient variations is different from that of the other two as reflected in the frequency curves of the coefficient in Figures 5 and 6 . This is because the two coatings follow more complicated mechanisms of acoustic energy loss, which is similar to the conversion from longitudinal waves to shear waves. Although the Alberich acoustic coating and the trumpet cavity-type coating have good sound absorption effect when Poisson's ratio is less than 0.499, the sound absorption performance decreases suddenly when Poisson's ratio increases from 0.499 to 0.4999 . The reason is shown in Table 2. When Poisson's ratio increases from 0.499 to 0.4999 , Poisson's ratio only increases by 0.0001 , but the Lamy constant does increase by an order of magnitude. Then, the sound absorption coefficient decreases suddenly.

According to Figures 5, 6, and 8, the sound absorption coefficient calculated by FEM using the static modulus is significantly lower than using the dynamic one, which is because the dynamic modulus is lower than the static one and the velocity of sound is lower accordingly. Additionally, coupled with the higher loss factor, the attenuation constant is reduced, which can be calculated using (7) and (8). Consequently, the average sound absorption coefficient increases.

\section{Poisson's Ratio Varying with Frequency}

As shown in Figure 2, a standing wave tube method was applied to measure the vertical incidence sound absorption coefficient of the trumpet cavity-type acoustic coating. In addition, the modulus of the nitrile rubber used for acoustic coating was measured (Figure 7) and inputted into the finite element model. Poisson's ratio was set to $0.496,0.497,0.498$, $0.499,0.4992,0.4994,0.4995,0.4996,0.4997$, and 0.4998, respectively. In the frequency range of $1000-5000 \mathrm{~Hz}$, the sound absorption coefficients of one-third octave range of the acoustic coating were obtained by means of test and 

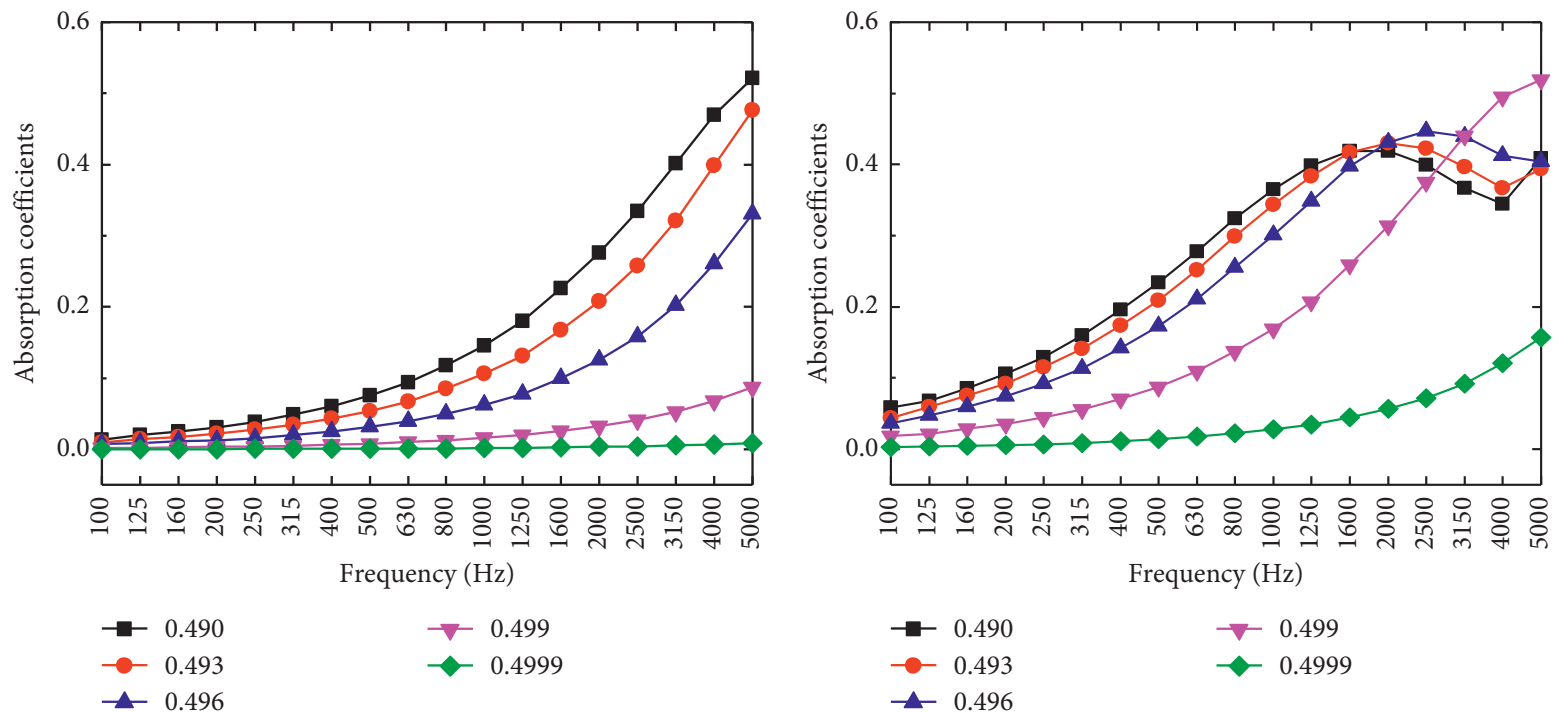

(a)

(b)

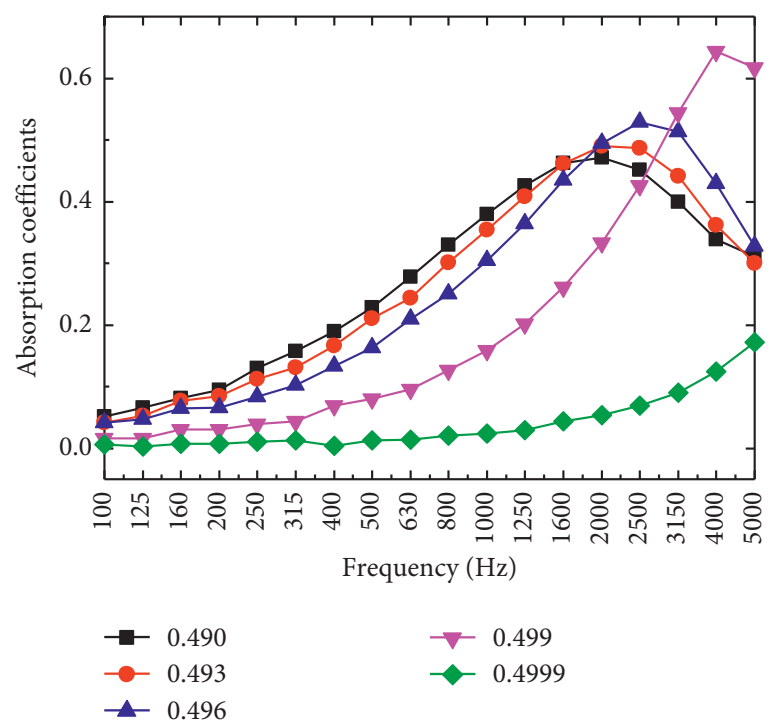

(c)

FIgURE 5: When the modulus shows no change with frequency, the sound absorption coefficient of the acoustic coating varies with Poisson's ratio: (a) homogeneous coating; (b) Alberich coating; (c) trumpet cavity-type coating.

calculation, as shown in Table 2 and Figure 9. According to the figure, when Poisson's ratio was set to the above values, the finite element calculation results of the sound absorption coefficient were starkly different from the measured results.

As indicated by the results of Ref. [12, 28], Poisson's ratio drops with the rise of frequency. According to this pattern and Table 1, a linear interpolation method was adopted to obtain Poisson's ratio which varies with frequency. Taking $1000 \mathrm{~Hz}$ as an example, the sound absorption coefficient measured is 0.420 , which is between the last two values of 0.442 and 0.336 in the first column of the coefficients shown in the table. The corresponding Poisson's ratio is 0.4997 and
0.4998, respectively. The linear interpolation was conducted to obtain Poisson's ratio of 0.49972 . The shaded numbers listed in the table represent the interval values of the interpolation. Varying within the frequency of $1000-5000 \mathrm{~Hz}$, the interpolated dynamic Poisson's ratios are presented in Figure 10. After linear fitting, they were found consistent with the characteristic as described in Ref. [12, 29]. More specifically, Poisson's ratio at low frequencies and the logarithmic frequency bears linear relationship.

With the interpolated dynamic Poisson's ratio inputted into the finite element model, the calculation results were obtained to indicate that the sound absorption coefficients 


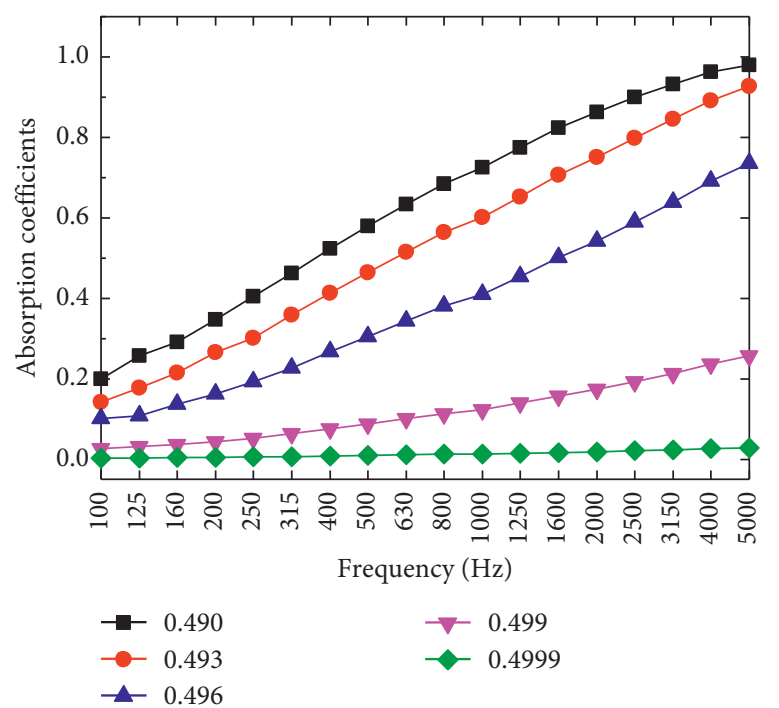

(a)

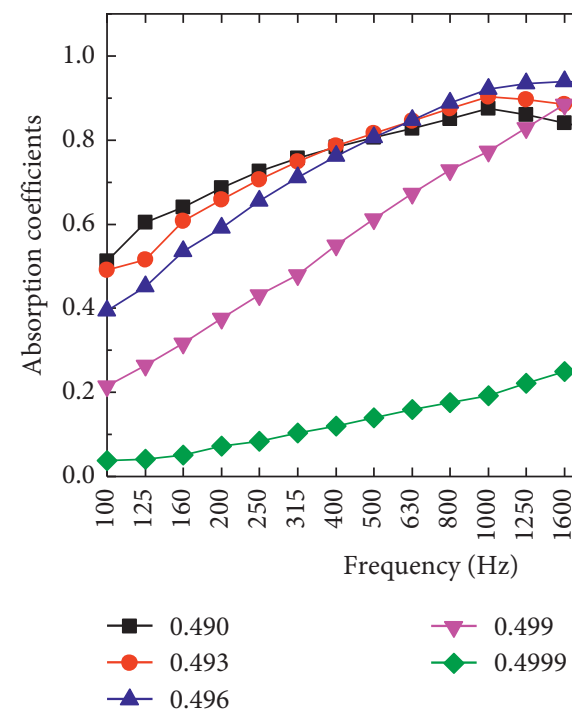

(c)

FIgURE 6: When the modulus varies with frequency, the sound absorption coefficient of the acoustic coating varies with Poisson's ratio: (a) homogeneous coating; (b) Alberich coating; (c) trumpet cavity-type coating.

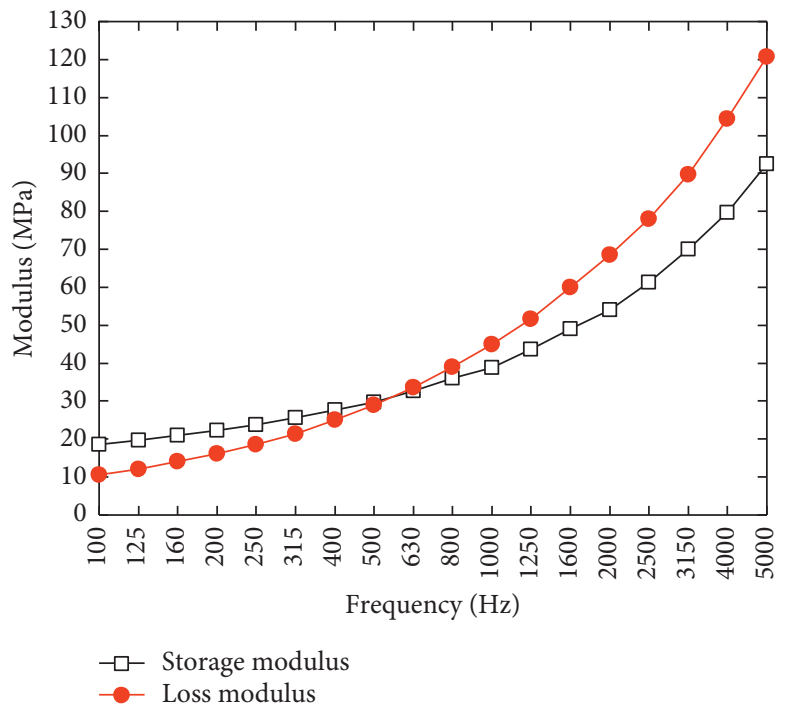

Figure 7: Dynamic modulus of carbon black filled nitrile rubber. 
TABle 1: Poisson's ratio, Lamy constant, and their rate of change.

\begin{tabular}{lccc}
\hline Poisson's ratio & Lamy constant & Change rate of Poisson's ratio & Change rate of Lamy constant \\
\hline 0.490 & $2.3 E+09$ & 0 & 0 \\
0.493 & $3.3 E+09$ & $0.61 \%$ & $43 \%$ \\
0.496 & $5.8 E+09$ & $1.22 \%$ & $152 \%$ \\
0.499 & $2.33 E+10$ & $1.84 \%$ & $912 \%$ \\
0.4999 & $2.33 E+11$ & $2.02 \%$ & $10034 \%$ \\
\hline
\end{tabular}

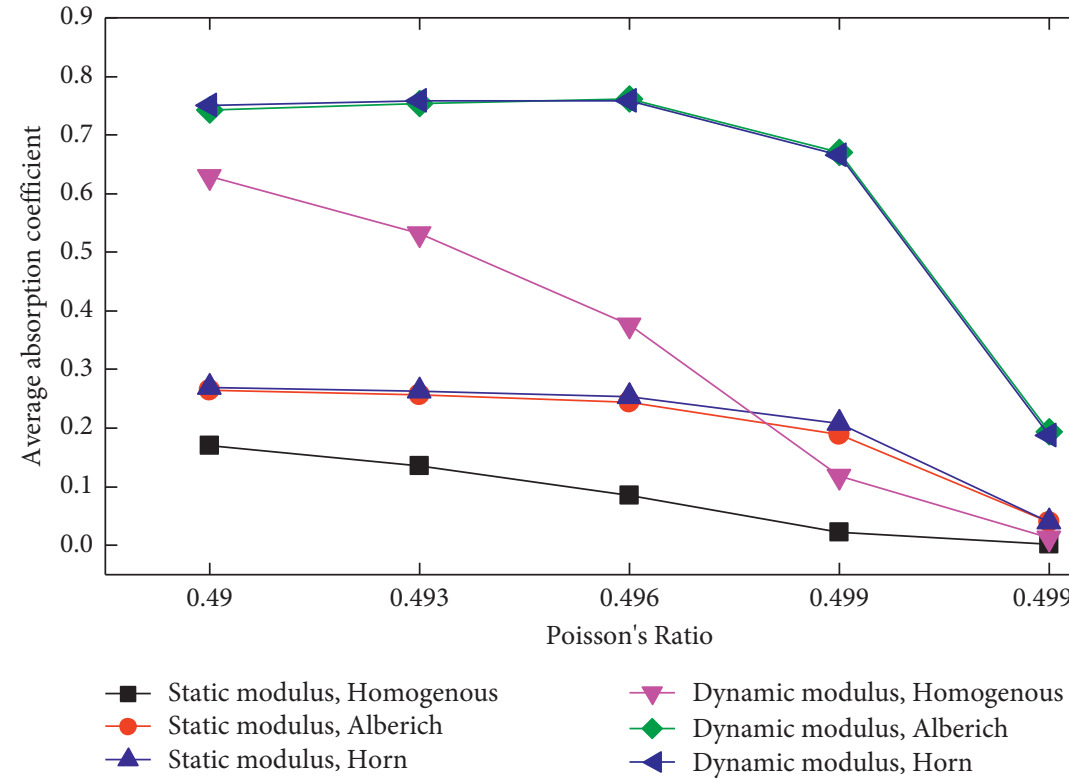

Figure 8: Variation of the average sound absorption coefficient with Poisson's ratio.

TABLE 2: Calculated and measured sound absorption coefficient.

\begin{tabular}{cccccccccc}
\hline \multicolumn{10}{c}{ Frequency } \\
\hline & Poisson's ratio & 1000 & 1250 & 1600 & 2000 & 2500 & 3150 & 4000 & 5000 \\
& 0.496 & 0.922 & 0.935 & 0.942 & 0.935 & 0.911 & 0.866 & 0.796 & 0.718 \\
& 0.497 & 0.917 & 0.939 & 0.957 & 0.960 & 0.946 & 0.909 & 0.841 & 0.756 \\
& 0.498 & 0.889 & 0.922 & 0.956 & 0.978 & 0.982 & 0.963 & 0.908 & 0.826 \\
Calculated & 0.499 & 0.772 & 0.821 & 0.879 & 0.930 & 0.969 & 0.996 & 0.994 & 0.953 \\
& 0.4992 & 0.720 & 0.769 & 0.831 & 0.888 & 0.937 & 0.978 & 0.997 & 0.979 \\
& 0.4994 & 0.642 & 0.692 & 0.756 & 0.817 & 0.874 & 0.929 & 0.973 & 0.985 \\
& 0.4995 & 0.589 & 0.638 & 0.701 & 0.764 & 0.823 & 0.884 & 0.940 & 0.970 \\
& 0.4996 & 0.524 & 0.570 & 0.631 & 0.692 & 0.752 & 0.816 & 0.883 & 0.930 \\
Measured & 0.4997 & 0.442 & 0.483 & 0.538 & 0.595 & 0.651 & 0.715 & 0.787 & 0.847 \\
& 0.4998 & 0.336 & 0.369 & 0.414 & 0.461 & 0.509 & 0.564 & 0.630 & 0.692 \\
& & 0.420 & 0.615 & 0.815 & 0.900 & 0.985 & 0.955 & 0.830 & 0.755 \\
\hline
\end{tabular}

were well consistent with the test results, as shown in Figure 9. The aforementioned dynamic Poisson's ratios were used to calculate the sound absorption characteristics of the Alberich coating made of the same nitrile rubber as the trumpet cavity-type coating. Figure 11 shows the calculation and measured results, suggesting that the sound absorption coefficients calculated using the static Poisson's ratios are significantly deviant from the test results, and that the ones calculated using the dynamic ratios are more consistent with the test results. In addition, it can be seen from the figure that dynamic Poisson's ratio should be used to obtain more accurate results for the finite element calculation of the 


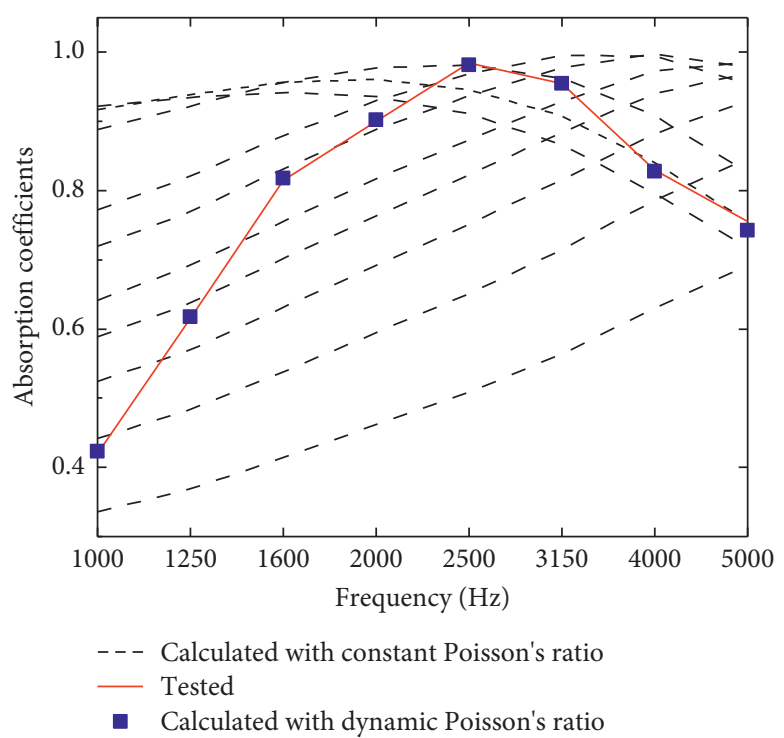

FIGURE 9: Calculation and measurement results of the sound absorption coefficient of the trumpet cavity-type acoustic coating.

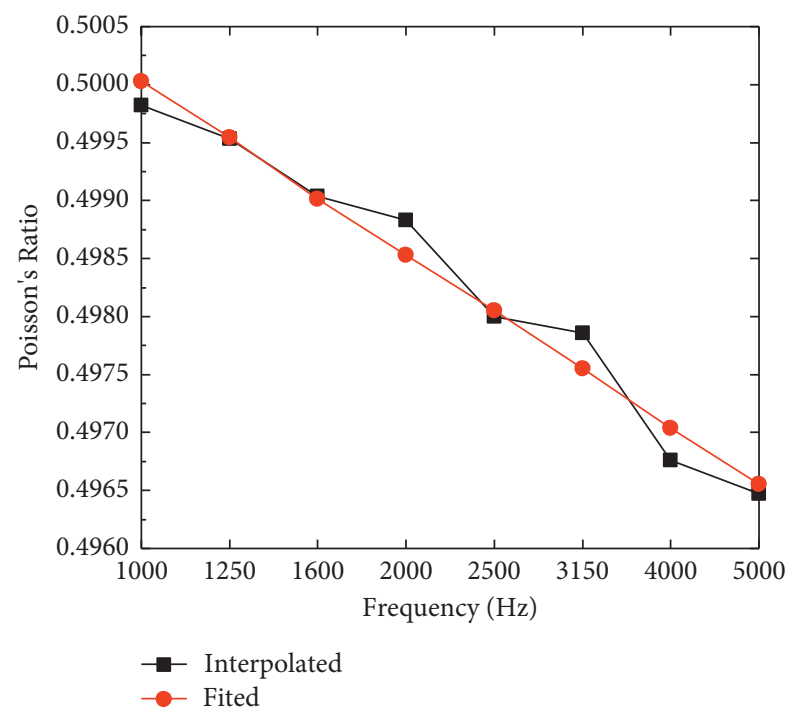

FIGURE 10: Interpolated Poisson's ratio varying with frequency.

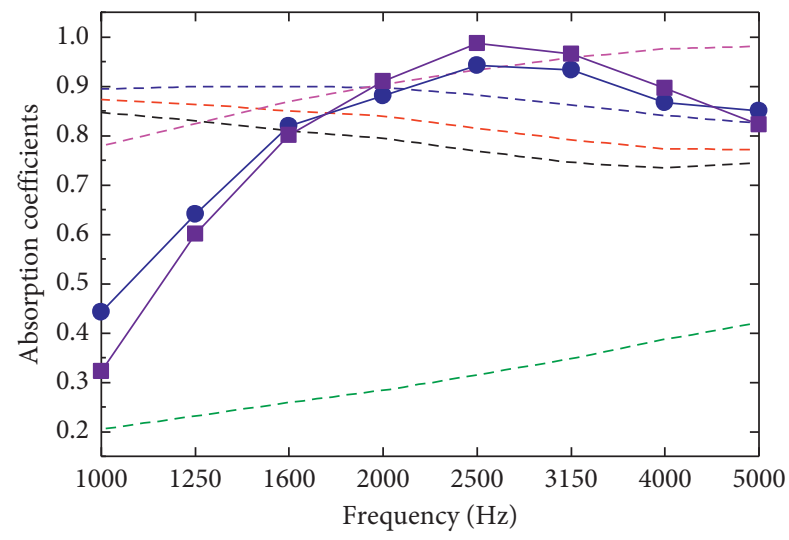

- - C Calculated with Poisson's Ratio $=0.490$

- - Calculated with Poisson's Ratio $=0.493$

- - - Calculated with Poisson's Ratio $=0.496$

- - - Calculated with Poisson's Ratio $=0.499$

- - Calculated with Poisson's Ratio $=0.4999$

- Calculated with dynamic Poisson's Ratio

$\rightarrow$ Measured

Figure 11: Calculation and measurement results of the sound absorption coefficients of the Alberich coating. 
sound absorption characteristics of acoustic coatings. Unfortunately, there is still no accurate and reliable method available to get the dynamic Poisson's ratios of viscoelastic materials [11].

\section{Conclusion}

(1) When Poisson's ratio of homogeneous acoustic coating, Alberich acoustic coating, and trumpet cavity-type coating varies from 0.49 to 0.4999 , the larger Poisson's ratio is, the larger the frequency of the first absorption peak is, the smaller the absorption coefficient is in the frequency range below the first absorption peak, and the smaller the average absorption coefficient is in the analysis frequency range.

(2) More accurate calculation results of sound absorption coefficient can be obtained by using Poisson's ratio varying with frequency. In the analysis of the sound absorption performance of acoustic coatings, the accurate dynamic Poisson's ratio with the change of frequency should be used.

(3) Poisson's ratio of viscoelastic materials varies in a very small numerical range; such a small change has a great influence on the sound absorption performance of acoustic coatings. Therefore, it is urgent to study the accurate method to obtain the dynamic Poisson's ratio.

\section{Data Availability}

The data used to support the findings of this study are available from the corresponding author upon request.

\section{Conflicts of Interest}

The authors declare that they have no conflicts of interest.

\section{Acknowledgments}

This work was financially supported by the Scientific Research Project of Education Department of Hunan Province, China (Project no. 19C0589).

\section{References}

[1] L. Alimonti and N. Atalla, "Efficient modeling of flat and homogeneous acoustic treatments for vibroacoustic finite element analysis. Finite size correction by image sources," Journal of Sound and Vibration, vol. 388, pp. 201-215, 2017.

[2] X. L. Yao, X. Z. Wang, F. Z. Pang, and L. Q. Sun, "Study on Acoustic Characteristics of Underwater Coating Structure," Noise and Vibration Control, vol. 30, no. 4, pp. 1-5, 2010.

[3] S. M. Ivansson, "Erratum:," Journal of the Acoustical Society of America, vol. 131, no. 4, p. 3180, 2012.

[4] S. M. Ivansson, "Numerical design of Alberich anechoic coatings with superellipsoidal cavities of mixed sizes," Journal of the Acoustical Society of America, vol. 123, no. 5, p. 3466, 2008.

[5] Z. Zhang, L. Li, Y. Huang, and Q. Huang, "Sound absorption performance of underwater anechoic coating in plane wave normal incidence condition," IOP Conference Series: Materials Science and Engineering, vol. 522, no. 1, Article ID 012001, 2019.

[6] H. Bai, Z. Zhan, J. Liu, and Z. Ren, "From local structure to overall performance: An overview on the design of an acoustic coating," Materials, vol. 12, no. 16, 2019.

[7] A. C. Hladkyhennion and J. N. Decarpigny, "Analysis of the scattering of a plane acoustic wave by a doubly periodic structure using the finite-element method," Journal of the Acoustical Society of America, vol. 88, 2005.

[8] T. Meng, "Simplified model for predicting acoustic performance of an underwater sound absorption coating," Journal of Vibration and Control, vol. 20, no. 3, pp. 339-354, 2014.

[9] L. M. Lu, J. H. Wen, H. G. Zhao, and X. S. Wen, "Dynamical mechanical property of viscoelastic materials and its effect on acoustic absorption of coating," Acta Physica Sinica, vol. 63, no. 15, 2014.

[10] Z. Wei, H. Hou, N. Gao, Y. Huang, and J. Yang, "Complex Young's modulus measurement by incident wave extracting in a thin resonant bar," Journal of the Acoustical Society of America, vol. 142, no. 6, pp. 3436-3442, 2017.

[11] N. W. Tschoegl, W. G. Knauss, and I. Emri, "Poisson's ratio in linear viscoelasticity - A critical review," Mechanics of TimeDependent Materials, vol. 6, no. 1, pp. 3-51, 2002.

[12] T. Pritz, "Frequency dependences of complex moduli and complex Poisson's ratio of real solid materials," Journal of Sound and Vibration, vol. 214, no. 1, pp. 83-104, 1998.

[13] J. Zhong, H. Zhao, H. Yang, J. Yin, and J. Wen, "Effect of Poisson's loss factor of rubbery material on underwater sound absorption of anechoic coatings," Journal of Sound and Vibration, vol. 424, pp. 293-301, 2018.

[14] H. Meng, J. Wen, H. Zhao, L. Lv, and X. Wen, "Analysis of absorption performances of anechoic layers with steel plate backing," Journal of the Acoustical Society of America, vol. 132, no. 1, pp. 69-75, 2012.

[15] V. Easwaran and M. L. Munjal, "Analysis of reflection characteristics of a normal incidence plane wave on resonant sound absorbers: A finite element approach," Journal of the Acoustical Society of America, vol. 93, no. 3, pp. 1308-1318, 1993.

[16] Z. Jin, Y. Yin, and B. Liu, "Equivalent modulus method for finite element simulation of the sound absorption of anechoic coating backed with orthogonally rib-stiffened plate," Journal of Sound and Vibration, vol. 366, pp. 357-371, 2016.

[17] L. M. Lü, J. H. Wen, H. Zhao, H. Meng, and X. S. Wen, "Lowfrequency acoustic absorption of viscoelastic coating with various shapes of scatterers," Wuli Xuebao/Acta Physica Sinica, vol. 61, 2012.

[18] J. Li and S. Li, "Topology optimization of anechoic coating for maximizing sound absorption," Journal of Vibration and Control, vol. 24, no. 11, pp. 2369-2385, 2018.

[19] C. Cai, K. C. Hung, and M. S. Khan, "Simulation-based analysis of acoustic absorbent lining subject to normal plane wave incidence," Journal of Sound and Vibration, vol. 291, no. 3-5, pp. 656-680, 2006.

[20] S. N. Panigrahi, C. S. Jog, and M. L. Munjal, "Multi-focus design of underwater noise control linings based on finite element analysis," Applied Acoustics, vol. 69, no. 12, pp. 1141-1153, 2008.

[21] H. Meng, J. Wen, H. Zhao, and X. Wen, "Optimization of locally resonant acoustic metamaterials on underwater sound absorption characteristics," Journal of Sound and Vibration, vol. 331, no. 20, pp. 4406-4416, 2012. 
[22] M. A. Lewniska, V. G. Kouznetsova, J. A. W. van Dommelen, A. O. Krushynska, and M. G. D. Geers, "The attenuation performance of locally resonant acoustic metamaterials based on generalised viscoelastic modelling," International Journal of Solids and Structures, vol. 126, pp. 163-174, 2017.

[23] A. C. Hladkyhennion and J. N. Decarpigny, "Analysis of the scattering of a plane acoustic-wave by a doubly periodic structure using the finite-element method- application to alberich anechoic coating," Journal of the Acoustical Society of America, vol. 90, no. 6, pp. 3356-3367, 1991.

[24] J. Villalobos-Luna, P. Lopez-Cruz, R. Ramirez-Valencia, and F. J. Elizondo-Garza, "Simulation of acoustic wave behavior in ducts and a plenum using finite elements package ANSYS," Journal of the Acoustical Society of America, vol. 112, no. 5, p. 2427, 2002.

[25] Y. Xu, G. Zhang, B. Zhou, H. Wang, and Q. Tang, "Analysis of acoustic radiation problems using the cell-based smoothed radial point interpolation method with Dirichlet-to-Neumann boundary condition," Engineering Analysis with Boundary Elements, vol. 108, no. Nov, pp. 447-458, 2019.

[26] Z. Zhang, L. Li, Y. Huang, and Q. Huang, "Sound absorption performance of underwater anechoic coating in plane wave normal incidence condition," in Proceedings of the 4th International Conference on Smart Material Research, ICSMR 2018, Sydney, Australia, November, 2018.

[27] Y. E. Hanfeng, M. Tao, and L. I. Junjie, "Sound absorption performance analysis of anechoic coatings under oblique incidence condition based on COMSOL," Journal of Vibration and Shock, vol. 038, no. 12, pp. 213-218, 2019.

[28] T. Pritz, "The Poisson's loss factor of solid viscoelastic materials," Journal of Sound and Vibration, vol. 306, no. 3-5, pp. 790-802, 2007.

[29] S. H. Sohrabi and M. J. Ketabdari, "Numerical simulation of a viscoelastic sound absorbent coating with a doubly periodic array of cavities," Cogent Engineering, vol. 5, no. 1, 2018. 